\title{
Até quando o Brasil será conhecido como o país da cesárea?
}

\section{Until when will Brazil be known as the country of cesarean section?}

Talvez a Obstetrícia seja a especialidade médica que mais se desenvolveu nos últimos 200 anos. A incorporação de grandes avanços científicos mudou completamente o panorama obstétrico e reduziu drasticamente a morbimortalidade materna e perinatal. Dentro desse contexto, a cesárea (CS) deixou de ser a forma de resolução da gestação utilizada apenas "na última hora", como tentativa de salvaguardar a vida da mãe ou do feto, para se tornar, em algumas situações de alto risco gestacional, a melhor opção de parto para o binômio. Como o procedimento foi se tornando mais seguro ao longo do tempo, os obstetras não tinham mais argumentos para retardar a indicação de resolução da gestação por via alta em situações de risco materno e/ou fetal. Adicionalmente, questões religiosas, a urbanização das populações, a valorização do feto após o advento do exame ecográfico e a hospitalização do parto colaboraram para o incremento mundial do número de $\mathrm{CSs}^{1}$.

A Organização Mundial de Saúde (OMS) declarou, em 1985, que nenhuma região deve ter taxas de CS superiores a $15 \%{ }^{2}$. Estudos que avaliaram a morbimortalidade materna e perinatal em pacientes submetidas à CS não encontraram reduções nesses indicadores quando as taxas foram superiores a $15 \% \%^{3}$. Por outro lado, as taxas mínimas recomendadas de CS necessárias para evitar a morbimortalidade materna e neonatal devem ser de 5 e $10 \%$, respectivamente ${ }^{4,5}$. No entanto, esse objetivo não parece ser muito exequível, tanto em países desenvolvidos como naqueles em desenvolvimento.

Em 2012, Gibbons et al. publicaram dados de uma pesquisa referente aos partos realizados em 137 países-membros da Organização das Nações Unidas (ONU), representando mais de $95 \%$ do número mundial de nascimentos no ano de $2008^{6}$. Nesse período, foram realizadas, aproximadamente, 18,5 milhões de CSs. Em 40\% dos países, as taxas foram inferiores a $10 \%$, em $10 \%$, entre 10 a $15 \%$, e, em cerca de $50 \%$, as cifras foram superiores a $15 \%$. Em 54 países (com taxas inferiores a 10\%), ocorreram $60 \%$ dos partos no mundo e foram feitas 4,5 milhões de CSs (25\% das taxas globais). Por outro lado, 13,5 milhões de CSs ( $73 \%$ das taxas globais) foram realizadas nos 69 países com taxas superiores a $15 \%$, locais onde ocorreram $37,5 \%$ do total de nascimentos. Levando em consideração a taxa ideal de CS entre 5 a $15 \%$, nessa pesquisa também foram publicados outros achados intrigantes, demonstrando que adicionais 3,2 milhões CSs deveriam ter sido realizadas nos 54 países com taxas $<10 \%$ (países na África e na Ásia, e Haiti). No entanto, 6,2 milhões de CSs foram

Correspondência

Alessandra Cristina Marcolin Departamento de Ginecologia e Obstetrícia Avenida Bandeirantes, 3.900

CEP: 14049-900

Ribeirão Preto (SP), Brasi

Recebido

16/06/14

Aceito com modificacõos

18/07/14

'Departamento de Ginecologia e Obstetrícia, Faculdade de Medicina de Ribeirão Preto, Universidade de São Paulo - USP Ribeirão Preto (SP), Brasil.

Conflito de interesses: não há 
realizadas desnecessariamente, sendo metade realizada na China (1.976.606) e no Brasil (960.687). O custo global das CSs “desnecessárias" foi estimado em cerca de US $\$ 2,3$ bilhões e o das CSs "necessárias" foi de, aproximadamente, US\$ 432 milhões. Finalmente, o dado mais alarmante: nos países onde existe a necessidade de maiores taxas de CS, o custo médio do procedimento foi avaliado em US\$135, enquanto o custo de uma CS nos países que as têm em excesso é de US\$373, o que significa que CSs são 2,8 vezes mais caras nesses locais. Cumpre destacar que, nesse trabalho de 2008, a taxa brasileira de CSs foi de 45,9\%, a maior entre todos os países avaliados e acima do percentual de $30 \%$ pactuado com o Ministério da Saúde do Brasil ${ }^{7}$.

$\mathrm{Na}$ atualidade, é possível inferir que as taxas de CS variam de acordo com a localidade, os tipos de serviços de saúde, as características da população atendida e os profissionais que prestam a assistência obstétrica. Todavia, outros fatores menos comentados colaboram para o incremento na realização desse procedimento: o temor materno da ansiedade e dor do trabalho de parto, a preocupação parental com o bem-estar fetal, a experiência negativa com partos anteriores, o fato de já ter sido submetida a uma CS sem complicações, a conveniência de um parto agendado para a equipe de saúde e a paciente, o medo de litígio pelos médicos e, especialmente, a percepção equivocada sobre os benefícios da $\mathrm{CS}^{8}$. Algumas evidências sugerem que a CS planejada, quando comparada ao parto vaginal (PV), pode reduzir o risco de desordens do assoalho pélvico materno, tais como incontinência urinária e fecal e prolapsos genitais, de morte fetal inesperada e de morbidade neonatal associada a prolapso de cordão, corioamnionite, sofrimento fetal agudo (SFA) e tocotraumatismos na apresentação pélvica. Embora a CS possa salvar a vida da mãe e/ou feto, em certos casos, o evidente aumento de seus percentuais não se encontra associado a reduções concomitantes na morbimortalidade materna e fetal, o que levanta a relevante preocupação de que ela seja realizada em demasia? .

Por outro lado, várias publicações têm fornecido informações importantes sobre a morbimortalidade materna e perinatal relacionada à $\mathrm{CS}$, reforçando a necessidade de discussão com a paciente sobre os riscos do procedimento e de se documentar a obtenção do termo de consentimento por escrito ${ }^{10}$. Liu e o Maternal Health Study Group of the Canadian Perinatal Surveillance System selecionaram uma coorte de 46.766 mulheres saudáveis, sem complicações obstétricas, que foram submetidas à primeira CS cuja indicação foi apresentação fetal pélvica (grupo CS eletiva), e as compararam com um grupo de 2.292.420 gestantes com características similares que tiveram indicação de indução do trabalho de parto (ITP). Em comparação com o PV, pacientes submetidas à CS eletiva tiveram risco maior de morbidade grave em geral —odds ratio $(\mathrm{OR})-(\mathrm{OR}=3,1)$, histerectomia $(\mathrm{OR}=3,2)$, hemorragia exigindo histerectomia $(\mathrm{OR}=2,1)$, complicação anestésica $(\mathrm{OR}=2,3)$, parada cardiorrespiratória $(\mathrm{OR}=5,1)$, tromboembolismo venoso $(\mathrm{OR}=2,2)$, infecção puerperal grave $(\mathrm{OR}=3,0)$, hematoma de parede $(\mathrm{OR}=5,1)$ e internação prolongada $(\mathrm{OR}=1,5)$. No entanto, mulheres que faziam parte do grupo de ITP mas tiveram suas gestações resolvidas por CS de urgência exibiram as maiores taxas de morbimortalidade dentre todas as gestantes avaliadas ${ }^{11}$. Além de resultados adversos maternos semelhantes aos citados, outros trabalhos também demonstraram maior risco de hemorragias, apontando a necessidade de hemotransfusão, rotura uterina, admissão da mãe em unidade de tratamento intensivo (UTI), readmissão hospitalar, problemas em gestações subsequentes (por exemplo, infertilidade, gestação ectópica, aborto e placenta prévia) e maiores custos ${ }^{12-14}$.

No aconselhamento sobre riscos, é crucial enfatizar que quanto maior o número de CSs anteriores, maior é o risco de complicações na gestação atual, como acretismo placentário, embolia amniótica, necessidade de hemotransfusão, histerectomia, lesões intestinais ou vesicais e admissão materna em UTI ${ }^{15}$. Em um estudo com 30.132 mulheres com CS prévia, a histerectomia foi necessária em $0,6,0,4,0,9,2,4,3,4$ e $8,9 \%$ das pacientes que eram submetidas a primeira, segunda, terceira, quarta, quinta, sexta ou mais CSs, respectivamente ${ }^{16}$.

CSs eletivas desnecessárias não aumentam apenas a morbimortalidade materna mas também a perinatal, quando comparadas ao PV espontâneo ${ }^{3,17}$. Resultados adversos neonatais incluem síndrome da angústia respiratória do recém-nascido $(\mathrm{RN})$, hipertensão pulmonar persistente, asfixia, atraso no desenvolvimento neurológico, admissão em UTI neonatal, tocotraumatismos, estabelecimento tardio da amamentação ${ }^{18}$. O risco de morte neonatal após a alta hospitalar permanece maior entre os RN de CS eletiva ${ }^{19}$. A primeira CS tem sido associada ao risco aumentado de prematuridade, baixo peso ao nascer, morte fetal e neonatal em gestações subsequentes, com implicações relevantes para a família, a sociedade e todo o sistema de saúde ${ }^{20}$.

Para que se entenda o quanto as CSs podem ser evitadas e sejam instituídas estratégias efetivas para reduzir as taxas de tal procedimento, é imprescindível saber por que elas são realizadas. Em 2010, Zhang et al. revisaram as indicações de CS, avaliando mais de 200.000 partos em 19 hospitais nos Estados Unidos, e demonstraram que $47.1 \%$ das CSs ocorreram devido à distócia, 30,9\%, por CS prévia, $27,1 \%$, por suspeita de SFA e $7,5 \%$, por apresentação pélvica. Vale a pena destacar um detalhe interessante desse estudo: $45 \%$ das CSs que ocorreram antes do início do trabalho de parto tinham como indicação CS prévia $^{21}$. Sendo assim, se o objetivo for reduzir as cifras de CS e suas complicações, é mandatório que a primeira CS seja evitada. Em 2011, Barber et al. mostraram que 
metade do aumento do número de CS se deu por causa do incremento das taxas de primeira CS e que as principais indicações para a sua realização foram, em ordem de frequência, distócia, suspeita de SFA, apresentação fetal anômala, gestação múltipla e suspeita de macrossomia fetal $^{22}$. Portanto, para evitar a primeira CS e reduzir globalmente as taxas desse tipo de parto, é preciso saber abordar essas indicações de maneira segura e diversa.

A primeira fase clínica do parto (período de dilatação) foi historicamente dividida por Friedman, na década de 1950 , em fase latente e fase ativa. Segundo esse pesquisador, a fase latente é a mais longa e tem duração de até 20 horas em primigestas e 14 horas em multíparas. $\mathrm{Na}$ fase ativa, que se inicia com $4 \mathrm{~cm}$ de dilatação, a velocidade de dilatação cervical aumenta significativamente, sendo possível estabelecer os diagnósticos de distócias nesse momento. A fase ativa prolongada é diagnosticada quando a dilatação cervical é progressiva, porém se dá em velocidade inferior a $1 \mathrm{~cm} /$ hora. Parada secundária da dilatação é definida como ausência de progressão da dilatação em dois exames cervicais com intervalo maior de duas horas, na presença de contrações uterinas adequadas ${ }^{23}$. No entanto, dados robustos e mais recentes do Consortium on Safe Labor têm estimulado discussões sobre a definição de progressão "normal" do trabalho de parto ${ }^{24}$. Nesse estudo, os autores demonstraram que, dos 4 aos $6 \mathrm{~cm}$, a velocidade da dilatação cervical independe da paridade da paciente, é mais lenta que a historicamente descrita e somente após os $6 \mathrm{~cm}$ a dilatação ocorre na velocidade relatada anteriormente. Sendo assim, eles sugerem que as distócias sejam diagnosticadas apenas a partir dos $6 \mathrm{~cm}$, quando então se iniciaria a fase ativa do trabalho de parto. Por isso, o American College of Obstetricians e Gynecologists (ACOG) sugeriu, em 2014, estratégias para redução de CSs adaptadas para essa nova realidade. Desde que as condições maternas e a vitalidade fetal sejam tranquilizadoras, uma fase latente prolongada deve ser manejada de maneira expectante, a dilatação cervical de $6 \mathrm{~cm}$ deve ser considerada limite para início da fase ativa e a CS fica reservada para mulheres com dilatação maior ou igual a esta, que já tenham corioamniorrexe, sem progressão da dilatação cervical com, pelo menos, 4 horas de contrações adequadas ou 6 horas de administração de ocitocina ${ }^{25}$.

Conceitos referentes ao período expulsivo também têm sido revisitados e questionados. Vários pesquisadores têm investigado a relação entre a duração desse período e os resultados maternos e neonatais adversos, na tentativa de definir o que pode ser considerado "normal". Com as melhorias na monitorização fetal intraparto, desfechos adversos em $\mathrm{RN}$ de primigestas não têm sido associados à duração do expulsivo ${ }^{26,27}$. Para multíparas, apesar de existirem poucos trabalhos na literatura, parece que resultados adversos neonatais só foram encontrados quando o período expulsivo foi superior a duas horas ${ }^{28}$. Por outro lado, complicações maternas e menor probabilidade de sucesso na via vaginal foram associadas ao prolongamento do expulsivo. No entanto, esses resultados adversos estão mais associados às ações e intervenções realizadas pelos profissionais que assistem a paciente do que à duração desse período por si só ${ }^{29}$. O ACOG sugere manter a tentativa de PV por, pelo menos, duas horas em gestantes multíparas e três horas em primigestas, se as condições maternas e fetais assim o permitirem. Períodos mais longos podem ser apropriados e adotados de forma individualizada, em pacientes analgesiadas e com fetos em apresentações anômalas, desde que a progressão da descida fetal seja documentada ${ }^{25}$.

Além da adoção de um tempo maior de observação do período expulsivo, duas outras condutas devem ser estimuladas visando à redução das taxas de CS: o PV instrumentalizado e a rotação manual da cabeça fetal. A comparação entre os resultados neonatais obtidos com o PV instrumentalizado e a CS realizada no período expulsivo não mostra diferença em relação à morbidade neonatal grave, representada por hemorragia intracraniana e morte ${ }^{30}$. Apenas $3 \%$ das mulheres submetidas à tentativa de parto fórcipe necessitarão de CS para resolução de sua gestação ${ }^{31}$. Todavia, na atualidade, orienta-se apenas a realização de fórcipe baixo (descida fetal em pelo menos +2 de De Lee) ou de alívio (polo cefálico visível no introito vaginal) por profissional treinado. Logo, treinar médicos residentes na realização de PV instrumentalizado utilizando simulação e estabelecer programas de educação continuada e reciclagem, para manutenção da qualidade na prática obstétrica, são atitudes que contribuirão para a redução segura das $\mathrm{CSs}^{25}$. Quando a cabeça fetal se encontra nas variedades de posições occipitais posteriores e transversas, situações em que o parto instrumentalizado não é mais indicado nos dias de hoje, a rotação manual do polo cefálico deve ser considerada antes que se opte pela $\mathrm{CS}^{32}$. Para que essa abordagem seja segura, a ultrassonografia intraparto é útil para aumentar a precisão do diagnóstico da variedade de posição nas situações de incerteza com o exame digital ${ }^{33}$.

A segunda indicação de CS mais comum é o diagnóstico intraparto de um traçado de frequência cardíaca fetal (FCF) anormal ou indeterminado. É crucial que haja uma abordagem padronizada capaz de estabelecer intervenções que identifiquem os resultados falso-positivos e reduzam as taxas de $\mathrm{CS}^{34}$. Traçados Categoria III ou anormais, que são representados por ausência de variabilidade na FCF associada a desacelerações tardias e/ou variáveis recorrentes ou bradicardia, estão relacionados ao comprometimento fetal e requerem imediata intervenção (manobras de reanimação fetal) para a melhora das condições fetais. Essas são constituídas por mudança de decúbito e hiperoxigenação materna, correção da hipotensão com expansão de volume 
(especialmente após analgesia de parto), correção da taquissistolia uterina com drogas $\beta$-miméticas, estímulo sonoro ou táctil do couro cabeludo fetal, retirada de agentes ocitócicos e amnioinfusão, caso haja corioamniorrexe. Entretanto, os traçados obtidos no período intraparto são classificados, em sua maioria, como Categoria II ou indeterminada, sendo, portanto, os responsáveis pelo maior número de indicações de CS devido à suspeita de SFA ${ }^{22}$. Essa categoria compreende uma ampla gama de padrões de FCF e, apesar de possuir um percentual maior de falso-positivos do que a Categoria III, também demanda avaliação criteriosa, vigilância contínua e instituição das manobras de reanimação, com posterior reavaliação ${ }^{35}$. Logo, é sensato que os profissionais, antes de optarem pela CS, se assegurem de que as medidas clínicas foram tomadas na tentativa de diagnosticar com maior precisão os fetos que realmente necessitam de nascimento imediato, para que, se assim for o caso, eles nasçam em melhores condições. O ACOG e outros autores recomendam ultimar o parto dentro dos próximos 30-60 minutos, por CS ou parto instrumentalizado quando possível, apenas se as manobras de reanimação fetal não melhoraram o padrão de FCF, ou seja, não o tornaram Categoria I (normal) ${ }^{34,36}$.

Apesar de as distócias e a suspeita de SFA agudo serem as principais indicações, outras menos comuns, em conjunto, são responsáveis por expressivo número de CS. Portanto, devem ser discutidas criteriosamente e abordadas de maneira diversa da atual, para que haja redução de seus percentuais. O PV na apresentação fetal pélvica tornou-se incomum a partir dos resultados do Term Breech Trial Study. Esse estudo demonstrou maiores taxas de morbimortalidade neonatal entre crianças nascidas de $\mathrm{PV}$, quando comparadas àquelas nascidas por $\mathrm{CS}^{37}$. Algumas sociedades apoiam a tentativa de PV na apresentação pélvica em candidatas selecionadas, em serviços com assistência obstétrica de qualidade e profissionais experientes ${ }^{38,39}$. Outra estratégia para a redução das taxas de CS nesse grupo de pacientes é a realização de versão externa na $36^{\mathrm{a}}$ semana de gestação, com analgesia locorregional ${ }^{25}$. Macrossomia fetal não é uma indicação de resolução da gestação, muito menos de CS. A fim de evitar morbimortalidade perinatal, especialmente tocotraumatismos, o ACOG recomenda que a CS deve ser limitada a situações em que o peso fetal é $\geq 5.000 \mathrm{~g} \mathrm{e} \geq 4.500 \mathrm{~g}$, em pacientes sem e com diabetes mellitus, respectivamente ${ }^{40}$. A prevalência de peso ao nascer superior a $5.000 \mathrm{~g}$ é muito baixa. Além disso, as pacientes devem ser esclarecidas sobre a imprecisão da ultrassonografia na determinação do peso fetal no último trimestre gestacional, conduta esta que deve ser desencorajada ${ }^{25}$.

A prevalência da gemelaridade vem aumentando nas últimas décadas, assim como a de CS por esse motivo. Nos Estados Unidos ${ }^{41}$, o percentual de CS entre as mulheres com gestações gemelares aumentou de $53 \%$, em 1995 , para $75 \%$, em 2008, principalmente por causa da ocorrência frequente de fetos em apresentação pélvica nessa condição. Quando o primeiro feto não é cefálico, não há controvérsias na literatura sobre o benefício da realização da CS ao feto/RN. No entanto, dados recuperados de um grande estudo randomizado, publicado em 2013, mostram que, quando o primeiro feto é cefálico, a CS não reduz os resultados adversos perinatais, independentemente da apresentação do segundo gemelar ${ }^{42}$. Portanto, a tentativa de PV deve ser estimulada nas apresentações fetais cefálica/cefálica e cefálica/pélvica, e, para que essa abordagem seja segura, é imprescindível o treinamento da equipe que assiste essas gestantes em PV pélvico e em ultrassonografia em sala de parto $^{25}$.

Promover maior acesso das mulheres a intervenções não médicas durante o trabalho de parto, ações desempenhadas por enfermeiras, fisioterapeutas e/ou doulas, como suporte contínuo e outras formas de alívio não farmacológico da dor, também pode contribuir para a redução das taxas de CS. Uma meta-análise publicada pela biblioteca Cochrane, em 2013, avaliou 12 ensaios (mais de 15.000 mulheres) e demonstrou que a presença do apoio contínuo "one-on-one" durante o trabalho de parto promoveu maior satisfação da paciente com a experiência e reduziu significativamente as taxas de $\mathrm{CS}^{43}$. Levando em consideração a ausência de danos mensuráveis associados, esse recurso é subutilizado no cenário nacional de assistência obstétrica. Outros métodos, como banhos, massagens, alternância de posição durante o trabalho de parto e eletroestimulação nervosa transcutânea, são eficazes no alívio da dor e parecem reduzir as taxas de CS, mas ainda há poucos trabalhos que demonstram claramente esse último benefício e os que foram publicados são muito variáveis em suas casuísticas e metodologias, dificultando comparações e análises ${ }^{44}$.

Esforços também devem ser direcionados para que haja redução das indicações de CS em situações de ITP, muito comuns nos dias de hoje. Em idades gestacionais inferiores a 41 semanas, a indução está indicada apenas se houver doença materna e/ou fetal que demande término da gestação. Por outro lado, em gestações com 41 semanas ou mais, a indução deve ser realizada, uma vez que reduz o risco de CS e de morbimortalidade perinatal ${ }^{45}$. Para aumentar o sucesso nessa indução, é necessária a interveniência de métodos de amadurecimento do colo uterino, sejam eles misoprostol, dinoprostone, balões ou laminária para dilatação cervical, sequenciais ou combinados, especialmente quando a cérvice é desfavorável ${ }^{46,47}$. Se as condições maternas e fetais permitirem, a instituição de duas estratégias na indução reduzirá as CSs por falha do procedimento: a adoção, como normal, de uma fase latente mais longa (até 24 horas) e a indicação da CS apenas após 
rotura de membranas e administração de ocitocina por, pelo menos, 12 horas $^{25}$.

Se não existirem iniciativas governamentais, institucionais e pessoais dos profissionais de saúde, não haverá redução nas taxas de CS. A utilização de um sistema de classificação dos tipos de CS que tenha a finalidade de monitorar e comparar as taxas entre os diferentes serviços é uma forma interessante de promover maior compreensão sobre a ocorrência desse tipo de parto e para que tipo de paciente ele é indicado. Um exemplo dessa classificação é a de Robson, que divide as gestantes em 10 grupos distintos, com base em suas histórias obstétricas (paridade, antecedente de CS, idade gestacional de resolução, se houve trabalho de parto, apresentação e número de fetos), independente da indicação da $\mathrm{CS}^{48}$. Visto que o sistema pode ser usado prospectivamente, na sua admissão hospitalar, cada mulher pode ser imediatamente classificada em um dos 10 grupos disponíveis. Vários estudos têm demonstrado a viabilidade do uso dessa classificação em hospitais públicos, privados e de ensino, e, quando utilizada de maneira contínua, tal ferramenta parece permitir a avaliação da assistência obstétrica e a implementação de estratégias direcionadas à redução das taxas de CS em grupos específicos ${ }^{49}$. Em adição, os gestores hospitalares devem providenciar a execução de auditorias e feedbacks sobre as CSs realizadas em seus serviços, garantir a confecção de protocolos de conduta e fornecer condições locais necessárias para que eles sejam cumpridos $^{25}$. Além disso, a atitude dos profissionais que assistem a paciente deve mudar. Eles devem estar abertos a discussões sobre o tema, à utilização da segunda opinião para indicação da CS e à mudança de sua conduta, o que implica em tentar o PV em paciente com antecedente de CS, por exemplo. É claro que todas essas mudanças de postura são reforçadas por pagamento justo dos serviços prestados pelos profissionais envolvidos com o parto, tanto do Sistema Único de Saúde (SUS) como da saúde suplementar. As organizações e instituições que norteiam a pesquisa nacional e internacional, assim como as agências de fomento, devem estimular e garantir a realização de estudos que proporcionem maior conhecimento e orientem a tomada de decisão mais segura em relação à CS.

Com relação à paciente, um amplo conjunto de abordagens multiprofissionais é necessário para se atingir menores taxas de CS. Melhorar o grau de instrução da paciente evitará CS. Gestantes com baixo nível educacional têm maior risco de chegar para atendimento médico em piores condições e ter piores resultados obstétricos ${ }^{50}$. Melhorias na qualidade do seguimento pré-natal, com intervenções que reduzam fatores de risco para complicações gestacionais, com controle mais adequado das doenças crônicas ou intercorrentes do ciclo gravídico, preparo corporal para o PV e suporte psicológico auxiliarão na obtenção de números aceitáveis de CS. É crucial que existam cursos voltados para a educação sobre o parto, que informem adequadamente a paciente e sua família sobre os riscos e benefícios maternos e perinatais da CS, que mudem a cultura "da cesárea" e que estimulem as pacientes a escolherem a melhor via de parto para a resolução de suas gestações, e não "a mais cômoda e fácil”. Como já dito, são vários os motivos que levam as gestantes a solicitarem a CS como modo de parto e que devem ser extensivamente trabalhados. Os profissionais de saúde precisam explorar as percepções erradas ou distorcidas sobre o PV com as mulheres e desenvolver estratégias que promovam e aumentem a confiança das gestantes sobre sua capacidade de dar à luz naturalmente.

\section{Referências}

1. Todman D. A history of caesarean section: from ancient world to the modern era. Aust N Z J Obstet Gynaecol. 2007;47(5):357-61 .

2. World Health Organization: Appropriate technology for birth. Lancet. 1985;2(8452):436-7.

3. Villar J, Valladares E, Wojdyla D, Zavaleta N, Carroli G, Velazco A, et al.; WHO 2005 global survey on maternal and perinatal health research group. Caesarean delivery rates and pregnancy outcomes: the $2005 \mathrm{WHO}$ global survey on maternal and perinatal health in Latin America. Lancet. 2006;367(9525):1819-29.

4. Dumont $A$, de Bernis L, Bouvier-Colle MH, Bréart G, MOMA study group. Caesarean section rate for maternal indication in sub-Saharan Africa: a systematic review. Lancet. 2001;358(9290):1328-33.

5. Betrán AP, Merialdi M, Laver JA, Bing-Shun W, Thomas J, Van Look $P$, et al. Rates of caesarean section: analysis of global, regional and national estimates. Paediatr Perinat Epidemiol. 2007;21 (2):98-1 13.
6. Gibbons L, Belizan JM, Laver JA, Betran AP, Merialdi M, Althabe $F$. Inequities in the use of cesarean section deliveries in the world. Am J Obstet Gynecol. 2012;206(4):33 1.e1-19.

7. Brasil. Ministério da Saúde. Portaria n 2.816, de 29 de maio de 1998 [Internet] [citado 2014 Jul 4]. Disponível em: <http:// dtr2001.saude.gov.br/sas/portarias/port98/GM/GM-2816.htm>

8. Hannah M, Whyte H, Hannah W, Hewson S, Amankwah K, Cheng $M$, et al.; Term Breech Trial Collaborative Group. Maternal outcomes at 2 years after planned cesarean section versus planned vaginal birth for breech presentation at term: the international randomized Term Breech Trial. Am J Obstet Gynecol. 2004;191(13):917-27.

9. Gregory KD, Jackson S, Korst L, Fridman M. Cesarean versus vaginal delivery: whose risks? Whose benefits? Am J Perinatol. 2012;29(1):7-18. 
10. Dresang LT, Leeman L. Cesarean Delivery. Prim Care. 2012;39(1):145-65.

11. Liu S, Liston RM, Joseph KS, Heaman M, Sauve R, Kramer MS; Maternal Health Study Group of the Canadian Perinatal Surveillance System. Maternal mortality and severe morbidity associated with low-risk planned cesarean delivery versus planned vaginal delivery at term. CMAJ. 2007;176(4):455-60.

12. Baskett TF, O'Connell CM. Severe obstetric maternal morbidity: a 15-year population-based study. J Obstet Gynaecol. 2005;25(1):7-9.

13. Deneux-Tharaux C, Carmona E, Bouvier-Colle MH, Bréart G. Postpartum maternal mortality and cesarean delivery. Obstet Gynecol. 2006;108(3 Pt 1):541-8.

14. Hofmeyr GJ, Barrett JF, Crowther CA. Planned cesarean section for women with a twin pregnancy. Cochrane Database Syst Rev. $2011 ;(12): C D 006553$.

15. Nisenblat V, Barak S, Griness OB, Degani S, Ohel G, Gonen R. Maternal complications associated with multiple cesarean deliveries. Obstet Gynecol. 2006; 108(1):21-6.

16. Silver RM, Landon MB, Rouse DJ, Leveno KJ, Spong CY, Thom EA, et al.; National Institute of Child Health and Human Development Maternal-Fetal Medicine Units Network. Maternal morbidity associated with multiple repeat cesarean deliveries. Obstet Gynecol. 2006; 107(6): 1226-32

17. MacDorman MF, Declercq E, Menacker F, Malloy MH. Infant and neonatal mortality for primary cesarean and vaginal births to women with "no indicated risk," United States, 1998-2001 birth cohorts. Birth. 2006;33(3):175-82.

18. Fogelson NS, Menard KM, Hulsey T, Ebeling M. Neonatal impact of elective repeat cesarean delivery at term: a comment on patient choice cesarean delivery. Am J Obstet Gynecol. 2005; 192(5):1433-6.

19. Villar J, Carroli G, Zavaleta N, Donner A, Wojdyla D, Faundes A, et al.; World Health Organization 2005 Global Survey on Maternal and Perinatal Health Research Group. Maternal and neonatal individual risks and benefits associated with caesarean delivery: multicentre prospective study. BM. 2007;335(7628): 1025.

20. Hemminki E, Shelley J, Gissler M. Mode of delivery and problems in subsequent births: a register-based study from Finland. Am J Obstet Gynecol. 2005;193(1):169-77.

21. Zhang J, Troendle J, Reddy U, Laughon SK, Branch DW, Burkman $\mathrm{R}$, et al.; Consortium on Safe Labor. Contemporary cesarean delivery practice in the United States. Am J Obstet Gynecol. 2010;203(4): 1-10.

22. Barber EL, Lundsberg LS, Belanger K, Pettker CM, Funai EF, Illuzzi $\mathrm{JL}$. Indications contributing to the increasing cesarean delivery rate. Obstet Gynecol. $2011 ; 118(1): 29-38$.

23. Friedman EA, Sachtleben MR. Amniotomy and the course of labor. Obstet Gynecol. 1963;22:755-70.

24. Zhang J, Landy HJ, Branch DW, Burkman R, Haberman S, Gregory $K D$, et al.; Consortium on Safe Labor. Contemporary patterns of spontaneous labor with normal neonatal outcomes: consortium on safe labor. Obstet Gynecol. 2010;1 16(6):1281-7.

25. American College of Obstetricians and Gynecologists; Society for Maternal-Fetal Medicine. Obstetric care consensus no. 1: safe prevention of the primary cesarean delivery. Obstet Gynecol. 2014;123(3):693-711.

26. Rouse DJ, Weiner SJ, Bloom SL, Varner MW, Spong CY, Ramin SM, et al.; Eunice Kennedy Shriver National Institute of Child Health and Human Development Maternal-Fetal Medicine Units Network. Second-stage labor duration in nulliparous women: relationship to maternal and perinatal outcomes. Am J Obstet Gynecol. 2009;201(14):357.e1-7.
27. Le Ray C, Audibert F, Goffinet F, Fraser W. When to stop pushing: effects of duration of second-stage expulsion efforts on maternal and neonatal outcomes in nulliparous women with epidural analgesia. Am J Obstet Gynecol. 2009;201(4):361 .e 1-7.

28. Allen VM, Baskett TF, O'Connell CM, McKeen D, Allen AC. Maternal and perinatal outcomes with increasing duration of the second stage of labor. Obstet Gynecol. 2009;1 13(6):1248-58.

29. Cheng YW, Shaffer BL, Bianco K, Caughey AB. Timing of operative vaginal delivery and associated perinatal outcomes in nulliparous women. J Matern Fetal Neonatal Med. $2011 ; 24(5): 692-7$.

30. Werner EF, Janevic TM, Illuzzi J, Funai EF, Savitz DA, Lipkind HS. Mode of delivery in nulliparous women and neonatal intracranial injury. Obstet Gynecol. 2011;118(6):1239-46.

31. O'Mahony F, Hofmeyr GJ, Menon V. Choice of instruments for assisted vaginal delivery. Cochrane Database Syst Rev. 2010;(1 1):CD005455.

32. Shaffer BL, Cheng YW, Vargas JE, Caughey AB. Manual rotation to reduce cesarean delivery in persistent occiput posterior or transverse position. J Matern Fetal Neonatal Med. 2011 ;24(1):65-72.

33. Chou MR, Kreiser D, Taslimi MM, Druzin ML, El-Sayed YY. Vaginal versus ultrasound examination of fetal occiput position during the second stage of labor. Am J Obstet Gynecol. 2004; 191 (2):521-4.

34. American College of Obstetricians and Gynecologists. Intrapartum fetal heart rate monitoring: nomenclature, interpretation, and general management principles; ACOG practice bulletin no. 106. Obstet Gynecol. 2009; 1 14(1):192-202.

35. Cahill AG, Roehl KA, Odibo AO, Macones GA. Association and prediction of neonatal acidemia. Am J Obstet Gynecol. 2012;207(3):206.e 1-8.

36. Miller DA. Intrapartum fetal heart rate monitoring: a standardized approach to management. Clin Obstet Gynecol. 201 1;54(1):22-7.

37. Hannah ME, Hannah WJ, Hewson SA, Hodnett ED, Saigal S, Willan AR. Planned caesarean section versus planned vaginal birth for breech presentation at term: a randomized multicenter trial. Lancet. 2000;356(9239): 1375-83.

38. ACOG Committee on Obstetric Practice. ACOG Committee Opinion No. 340. Mode of term singleton breech delivery. Obstet Gynecol. 2006;108(1):235-7.

39. Kotaska A, Menticoglou S, Gagnon R, Farine D, Basso M, Bos H, et al.; Society of Obstetricians and Gynaecologists of Canada. SOGC clinical practice guideline: vaginal delivery of breech presentation. Int J Gynaecol Obstet. 2009;107(2): 169-76.

40. Chatfield J. ACOG issues guidelines on fetal macrosomia. American College of Obstetricians and Gynecologists. Am Fam Physician. $2001 ; 64(1): 169-70$.

41. Lee HC, Gould JB, Boscardin WJ, El-Sayed YY, Blumenfeld YJ. Trends in cesarean delivery for twin births in the United States: 1995-2008. Obstet Gynecol. 2011;118(5):1095-101.

42. Barrett JF, Hannah ME, Hutton EK, Willan AR, Allen AC, Armson BA, et al.; Twin Birth Study Collaborative Group. A randomized trial of planned cesarean or vaginal delivery for twin pregnancy: twin birth study collaborative group. $N$ Engl J Med. 2013;369(14):1295-305.

43. Hodnett ED, Gates S, Hofmeyr GJ, Sakala C. Continuous support for women during childbirth. Cochrane Database Syst Rev. 2013;7:CD003766.

44. Simkin PP, $\mathrm{O}^{\prime}$ hara $M$. Nonpharmacologic relief of pain during labor: systematic reviews of five methods. Am J Obstet Gynecol. 2002;186(5 Suppl Nature):S131-59. 
45. Gülmezoglu AM, Crowther CA, Middleton P, Heatley E. Induction of labor for improving birth outcomes for women at or beyond term. Cochrane Database Syst Rev. 2012;6:CD004945.

46. Jozwiak M, Bloemenkamp KW, Kelly AJ, Mol BW, Irion O, Boulvain M. Mechanical methods for induction of labor. Cochrane Database Syst Rev. 2012;3:CD001233.

47. Hofmeyr GJ, Gülmezoglu AM, Pileggi C. Vaginal misoprostol for cervical ripening and induction of labor. Cochrane Database Syst Rev. 2010;(10):CD000941.
48. Robson MS. Can we reduce the caesarean section rate? Best Pract Res Clin Obstet Gynaecol. 2001;15(1):179-94.

49. Robson M, Hartigan L, Murphy M. Methods of achieving and maintaining an appropriate caesarean section rate. Best Pract Res Clin Obstet Gynaecol. 2013;27(2):297-308.

50. Tunçalp Ö, Souza JP, Hindin M, Santos CA, Oliveira TH, Vogel JP, et al.; WHO Multicountry Survey on Maternal and Newborn Health Research Network. Education and severe maternal outcomes in developing countries: a multicountry cross-sectional survey. BJOG. 2014;121(Suppl 1):57-65. 